\title{
INFLUENCIA DEL PASTOREO EN LA CUBIERTA VEGETAL Y LA GEOMORFODINÁMICA EN EL TRANSECTO DEPRESIÓN DEL EBRO-PIRINEOS ${ }^{(1)}$
}

\author{
Ries, J.B. ${ }^{(*)}$, Seeger, M. ${ }^{(*)}$, Marzolff, I. ${ }^{(*)}$ \\ (*) Physische Geographie, FB Geographie/Geowissenschaften, Campus II, Universität \\ Trier, D-54286 Trier \\ $\left.{ }^{(*}\right)$ Institut für Physische Geographie, Johann Wolfgang Goethe Universität \\ Frankfurt, Senckenberganlage 36, D-65235 Frankfurt/Main
}

\begin{abstract}
Resumen: El pastoreo extensivo de campos en barbecho o abandonados entre la Depresión Central del Ebro y el Pirineo Central impide la regeneración de la cubierta vegetal. Se desarrolla un patrón típico de areas con geomorfodinámica muy activa en los senderos del ganado, junto a otras extensas superficies estables. En el Prepirineo y el Pirineo Central, se puede incluso observar la reducción de la cubierta de matorral ya existente, de forma simultánea al incremento de la geomorfodinámica. Las zonas afectadas por fuerte erosión lineal aumentan a lo largo de los senderos, ya que éstos muestran tasas de escorrentía superficial y de erosión muy altas. A causa de estos efectos, los incentivos comunitarios a la ganadería ovina han de ser revisados de forma crítica.
\end{abstract}

Palabras clave: Abandono de cultivos, ganadería sedentaria y trashumante, sucesión de vegetación, geomorfodinámica, Depresión Central del Ebro, Prepirineo, Pirineo Central.

\begin{abstract}
Extensive grazing by sheep and goats on fallow land and abandoned fields in the Ebro basin and the Pyrenees impedes the regeneration of vegetation cover by browsing and hoof threads. An association of characteristic patterns of geomorphodynamically highly dynamic animal trails and largely stable areas develops. In the High Pyrenees and the Pre-Pyrenees, even existing matorral scrubs shows clear sign of thinning, while morphodynamic activity increases. Areas affected by erosion rills expand especially where sheep trails encourage their development by very high runoff and erosion rates. Regarding their effects on vegetation cover development and geomorphodynamic processes, sheep breeding subsidizing programmes by the European Union have to be critically assessed.
\end{abstract}

Key words: Land abandonment, grazing, vegetation succession, geomorphodynamics, Central Ebro Basin, Pre-Pyrenees, Central Pyrenees.

(1) Los mapas de este artículo están incluictos en el CD del final e este ejemplar. 


\section{Introducción}

Durante siglos los cultivos del Pirineo Central situados entre los 800 y $1500 \mathrm{~m}$ han servido, tras ser cosechados, como pasto para el ganado en trashumancia que descendía de los puertos para hibernar en el Valle del Ebro. En la Depresión del Ebro, los rebaños pastaban en los campos de secano, abonandolos a su vez. Cuando en mayo emprendían su regreso a los puertos de alta montaña paraban, preferentemente, en los pastos del Prepirineo a una altura entre 700 y 1200 m (García-Ruiz \& Lasanta, 1993).

Este sistema tradicional ganadero, típico de las regiones montañosas de la Península Ibérica, desapareció a finales del siglo XIX a causá de la despoblación y la consiguiente destrucción de la estructura agrícola tradicional. Por ejemplo, el municipio de Borau contaba a principios del siglo pasado con 5000 cabezas de ganado bovino, que llegaron a 3000 a mediados de los años 50, reduciéndose a tan sólo 500 cabezas en 1989. Desde comienzos de los años 90 las subvenciones al ovino, de $26,4 €$ por cabeza y año, por parte de la $\mathrm{CE}$ han causado un fuerte incremento del ganado trashumante, que en 1996 llegó a contar con 3000 cabezas (Lucht, 1998) y en la actualidad ronda los 4000 animales (información proporcionada por los ganaderos de la zona), con lo cual ya casi ha vuelto a alcanzar el máximo de principios del siglo pasado.

La recuperación de la trashumancia se debe por un lado a la creciente demanda en Europa de carne de ovino criada en condiciones "naturales", por el fuerte incremento de campos retirados en la Depresión Central del Ebro y en el Prepirineo y además por la creación de suficientes infraestructuras que permiten al pastor la vuelta a casa por la noche. Los animales, a su vez, vuelven a utilizar las antiguas cañadas. Además, se ha incrementado el número de pequeños rebaños no trashumantes, que aumentan así la presión ganadera. Por todo ello, se puede observar un sistema denso de pequeños senderos en las zonas pastoreadas y los consecuentes daños en prácticamente todas las plantas leñosas.

España contaba en el año 2001 con 23,3 millones de ovejas y 2,6 millones de cabras, que suponen aproximadamente el $25 \%$ del ganado ovino y caprino, respectivamente, de la Unión Europea y, precisamente, en el caso del ganado caprino se puede observar un incremento de la producción (Weiler \& Poschacher, 2002).

La evolución de la cubierta vegetal y su influencia en la generación de escorrentía superficial y erosión de suelo ha sido investigada en numerosos trabajos durante los ultimos 15 años (Sala, 1991; García-Ruiz \& Lasanta, 1993; Gallart et al., 1994; Llorens et al., 1997; García-Ruiz \& Valero-Garcés, 1998; Marzolff, 1999; Seeger, 2001; Ries, 2002), en los que se ha demostrado que, con la creciente antigüedad del abandono, los procesos geomorfodinámicos no decrecen como habría de suponer bajo condiciones de sucesión progresiva. 


\section{1. Áreas estudiadas y métodología}

\section{1. Áreas de estudio}

Las investigaciones se realizaron enmarcadas en el proyecto EPRODESERT (Ries et al., 1997) entre primavera de 1995 y otoño de 2002. Las áreas de estudio (Fig. 1) se localizan en María de Huerva, Depresión Central del Ebro semiárida $(<300 \mathrm{~mm}$ de precipitatión anuales), en Sabayés, al Norte de Huesca, en la vertiente sur del Prepirineo semiárido-subhúmedo $(670 \mathrm{~mm})$ y en Arnás, en el Pirineo Central subhúmedo $(980 \mathrm{~mm})$. En este transecto están representados los pastos invernales e intermedios del Nordeste español.

La vegetación climácica del área de estudio de Maria de Huerva, sabinares abiertos con un estrato herbáceo-arbustivo de escasa cobertura (el Rhamneto-Cocciferetum thuriferetosum) ha desaparecido casi completamente de la Depresión del Ebro a causa de

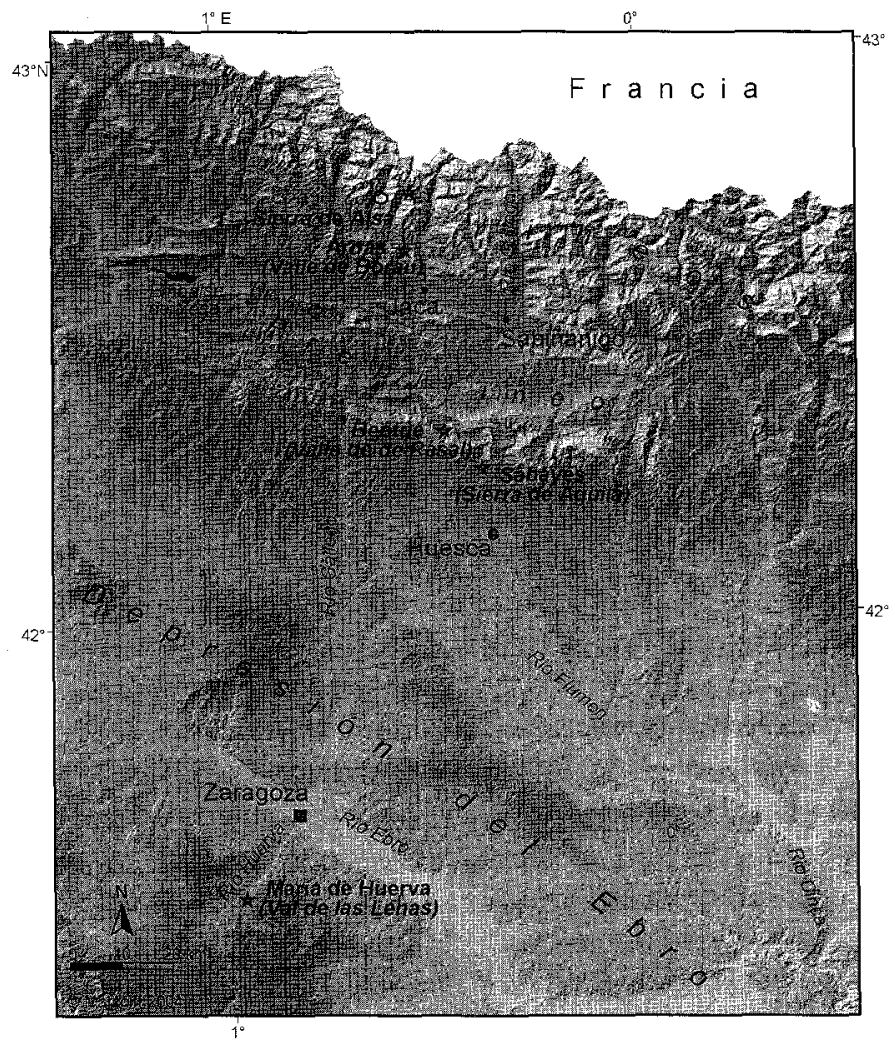

Figura 1. Localización de las zonas de estudio. 
la explotación intensiva de la zona durante siglos. El paisaje está dominado en la actualidad por las especies del estrato herbáceo-arbustivo xerófilo (Lygeum spartum, Artemisia berba-alba, Brachypodium ramosum) y las especies aromáticas (Thymus spec.) de los tomillares y albardinares (Braun-Blanquet \& de Bolós, 1987; Ibarra-Benlloch \& Martínez-Ferrer, 1995). Su cobertura oscila entre 10 y $25 \%$ en las solanas y 25 a $80 \%$ en las umbrías (Harrer, 1996). Sobre los Gypsisoles poco productivos se encuentran cultivos de secano de escaso rendimiento. En el barbecho la capacidad de infiltración se ve reducida por la generación de costras superficiales y la colonización por líquenes. El resultado es una generación de escorrentía superior a la de los campos labrados, y en consecuencia una inferior recarga del suelo con agua. El déficit hídrico generado de esa manera reduce notablemente la velocidad de la recolonización vegetal. Ambos factores aumentan el riesgo de erosión de suelo y son la causa de procesos de degradación del mismo (Ries et al., 2000; Seeger, 2001). De forma paralela a la retirada de tierras se ha incrementado la cantidad de ganado ovino y caprino. A mediados de los años 90 se contaban en el municipio de María de Huerva 3300 cabezas, cifra superior a la del máximo registrado en 1941 (Harrer, 1996). A causa del incremento de los rebaños trashumantes se ha de contar con un aumento de la presión ganadera en los proximos años.

El área de estudio de Sabayés pertenece a la transición entre los pisos meso y oromediterráneos, caracterizada por un bosque esclerófilo mediterráneo con Quercus rotundifolia y Quercus ilex como especies dominantes sobre suelos con notables acumulaciones de carbonatos que pueden clasificarse en áreas extensas como Calcisoles hipercálcicos. La vegetación actual es el resultado de una explotación maderera y ganadera secular (Montserrat i Martí, 1986) y está caracterizada por ejemplares muy degradados de porte arbustivo de Quercus rotundifolia y un matorral denso compuesto por aliaga (Genista scorpius), boj (Buxus sempervirens) y coscoja (Quercus coccifera).

En los ultimos años, los rebaños trashumantes se detienen en el Prepirineo, durante su camino primaveral hacia los puertos, dejando una red densa de pequeños senderos que recorre las laderas y provoca el derrumbe de las antiguas terrazas de los cultivos.

El área de estudio de Arnás está situada en el Pirineo Central, dentro del límite superior del piso montano submediterráneo. Su vegetación está compuesta por especies arbóreas pubescentes (Quercus cerrioides), en transición a un bosque dominado por coníferas (Pinus syluestris) (Rivas Martínez, 1987). Tras siglos de explotación maderera, agrícola y ganadera no se pueden encontrar restos de la asociación climácica de Buxo-Quercetum pubescentis. Tras el abandono de la actividad tradicional, las terrazas están siendo colonizadas por aliagas acompañadas por herbáceas como Brabypodium spec.. La colonización progresiva incluye arbustos como el boj, el espino albar (Crataegus monogyna) para llegar finalmente el enebro (Juniperus communis) y el pino silvestre. Los suelos, de drenaje deficiente, están cubiertos por acumulaciones de esqueleto edáfico, testigo de la erosión sufrida, y en amplias zonas antiguamente cultivadas pueden clasificarse como regosoles estágnicos. Los valores 


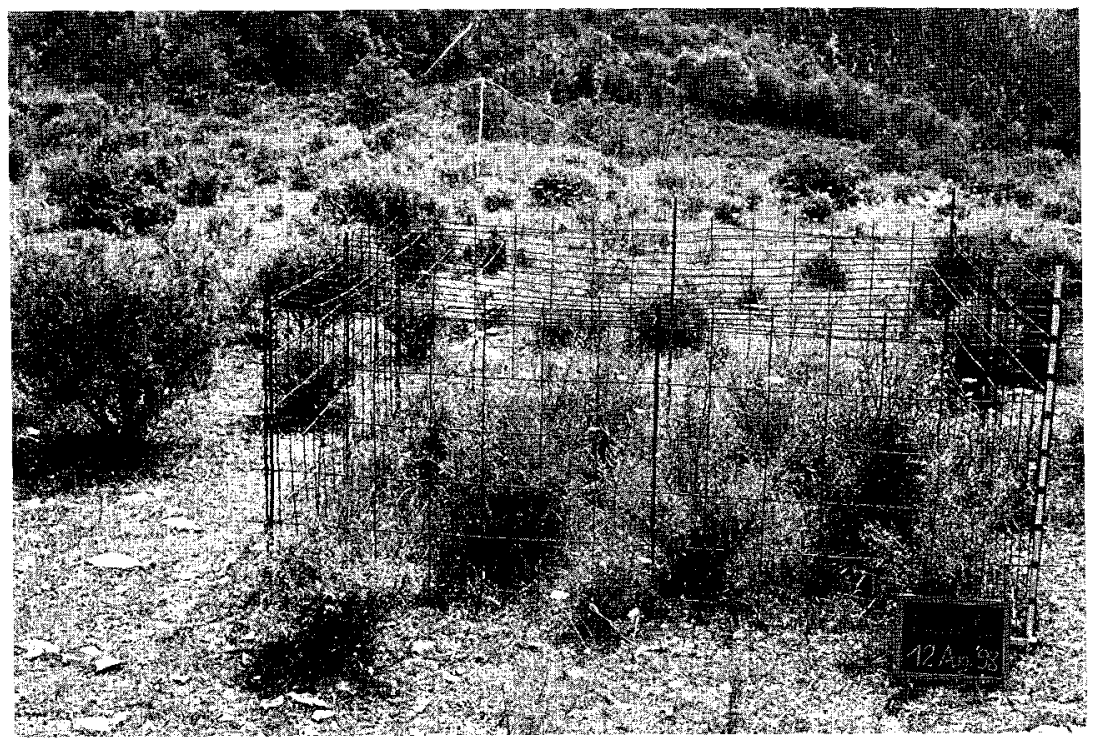

Figura 2. Parcela de control (parcela de exclusión de ganado) destinada al análisis de la influencia del pastoreo en la zona de estudio de Arnás, Pirineo Central (Foto tomada 2 años después de su instalación).

de escorrentía varían entre 0 y $57 \%$ de la precipitación aplicada, con una media de $19 \%$ relativamente alta, si se tiene en cuenta que la cubierta vegetal es densa. Las tasas de erosión medias son en cambio relativamente bajas y solo alcanzan $5 \mathrm{~g} \mathrm{~m}^{-2}$.

\subsection{Métodos}

La evolución de la cubierta vegetal y de la geomorfodinámica se ha analizado detalladamente utilizando áreas-test de $36 \times 24 \mathrm{~m}$. Para ello, la cubierta vegetal y la geomorfodinámica fueron analizadas en varios momentos, utilizando una resolución de $50 \times 50 \mathrm{~cm}$ y divididas en cinco clases: desde sin vegetación $(0-5 \%)$ hasta cubierta cerrada (>90-100\%) y desde "sin erosión" hasta "erosión lineal". Los mapas de retículas de ambos factores se han analizado con un SIG, y así se han podido generar mapas de cambios para cada uno de los factores que se han tenido en cuenta, que varían entre incremento y reducción "leve", "medio" y "fuerte". Los patrones de densidad e intensidad de los cambios muestran la distribucion espacial de las diferentes tendencias. A poca distancia de las parcelas se instalaron jaulas de control del pastoreo $\left(4 \mathrm{~m}^{2}\right)$, el las cuales se puede evaluar la influencia del ganado (Fig. 2). A través de muestreos sucesivos de esta parcela y de otra de referencia sin protección se cuantifica la sucesión vegetal con y sin pastoreo (Fig. 7). Además, se determinaron con un 
simulador de lluvia móvil los coeficientes de generación de escorrentía y la producción de sedimentos en diferentes ambientes. Para ello se aplicó una intensidad de precipitación simulada de $40 \mathrm{~mm} \mathrm{~h}^{-1}$ durante $30 \mathrm{mn}$ (Ries et al., 2000).

\section{Resultados}

\subsection{Depresión Central del Ebro.}

La parcela de la Depresión Central del Ebro, situada en el bajo valle del Huerva, afluente ibérico del Ebro, muestra la evolución de un bancal cultivado, abandonado hace 60 años y desde entonces pastoreado. La parcela experimental se caracteriza por una alta variabilidad de cubrimiento vegetal y de clases geomorfodinámicas, que representan las islas de vegetación y los correpondientes senderos producidos por los animales. Estos senderos destacan especialmente a lo largo de los bordes de los aterrazamientos y en la cabecera del gully que se instala en la parcela estudiada. En los límites de las terrazas se observa 'erosión laminar intensa' y 'erosión lineal'. La cubierta vegetal a comienzo de los muestreos es sorprendentemente baja, con tan solo un $26 \%$ ce cubrimiento (Tabla 1). A causa de las abundantes precipitaciones durante el periodo siguiente, la cubierta vegetal aumenta hasta alcanzar una media de $46 \%$. A pesar de este 'fuerte incremento' no se produce un descenso análogo de la actividad geomorfodinámica (Tabla 2). Al contrario, se puede observar incluso un aumento en las clases 'erosión laminar fuerte' y 'erosión lineal', donde la cubierta vegetal es baja. Los bordes de los bancales son especialmente activos en este sentido a causa del pisoteo concentrado del ganado.

Tabla 1. Distribución de clases de cobertura vegetal en \% de superficie y media ponderada en las parcelas de María de Huerva, Sabayés y Arnás. CV = cubierta vegetal.

\begin{tabular}{|c|c|c|c|c|c|c|}
\hline $\begin{array}{l}\text { muestreo de } \\
\text { vegetación }\end{array}$ & $\begin{array}{c}\text { Sin vegetación } \\
(0-5 \%) \\
{[\%]} \\
\end{array}$ & $\begin{array}{c}\text { CV escasa } \\
(>5-30 \%) \\
{[\%]}\end{array}$ & $\begin{array}{c}\text { CV discontinua } \\
(>30-60 \%) \\
{[\%]}\end{array}$ & $\begin{array}{c}\text { CV densa } \\
(>60-90 \%) \\
{[\%]}\end{array}$ & $\begin{array}{c}\text { CV cerrada } \\
(>90-100 \%) \\
{[\%]}\end{array}$ & $\begin{array}{c}\text { Cubimiento medio } \\
\text { ponderacio } \\
{[\%]}\end{array}$ \\
\hline \multicolumn{7}{|c|}{ María de Huerva } \\
\hline Mayo 95 & 14,3 & 24,0 & 20,8 & 13,0 & 2,6 & 26,1 \\
\hline Abril 98 & 3,8 & 9,7 & 15,4 & 25,0 & 9,7 & 46,2 \\
\hline \multicolumn{7}{|c|}{ Sabayés } \\
\hline Mayó 95 & 0,1 & 4,4 & 13,9 & 55,7 & 25,8 & 73,3 \\
\hline Abril 98 & 0,1 & 16,2 & 30,2 & 30,8 & 22,6 & 60,6 \\
\hline \multicolumn{7}{|c|}{ Arnás } \\
\hline Junio 95 & 0,0 & 0,8 & 17,2 & 56,7 & 25,3 & 74,4 \\
\hline Ago. 98 & 0,0 & 1,8 & 33,4 & 35,9 & 28,9 & 69,7 \\
\hline
\end{tabular}




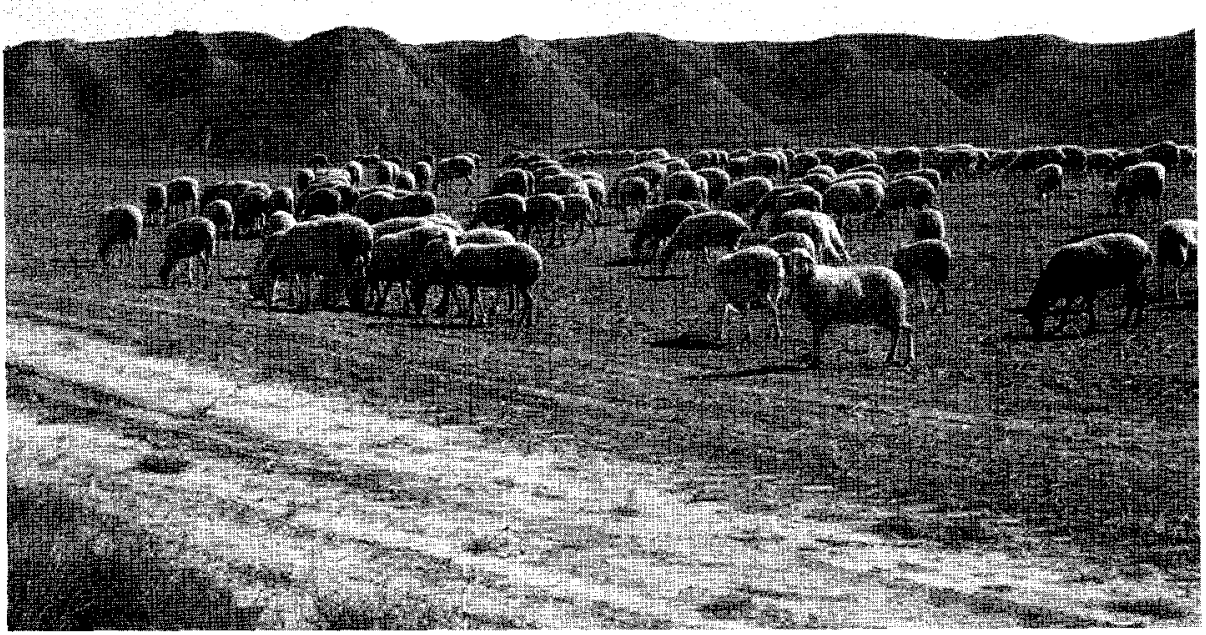

Figura 3. Rebaño trashumante en un cultivo cosechado en la zona de María de Huerva, Depresión Central del Ebro.

Tabla 2. Distribución de clases de geomorfodinámica actual en \% de la superficie de la parcelas de María de Huerva, Sabayés y Arnas 1995 y 1998.

\begin{tabular}{|c|c|c|c|c|c|}
\hline $\begin{array}{l}\text { muestreo de } \\
\text { geomorfo- } \\
\text { dinámica }\end{array}$ & $\begin{array}{c}\text { Erosión } \\
\text { nula } \\
{[\%]}\end{array}$ & $\begin{array}{c}\text { Erosión } \\
\text { laminar leve } \\
{[\%]}\end{array}$ & $\begin{array}{c}\text { Erosión } \\
\text { laminar intensa } \\
{[\%]}\end{array}$ & $\begin{array}{c}\text { Erosión } \\
\text { lineal } \\
{[\%]}\end{array}$ & $\begin{array}{c}\text { Acumulación } \\
{[\%]}\end{array}$ \\
\hline \multicolumn{6}{|c|}{ María de Huerva } \\
\hline Mayo 95 & 27,6 & 33,4 & 6,1 & 4,3 & 3,3 \\
\hline Abril 98 & 24,2 & 31,5 & 9,0 & 4,7 & 4,1 \\
\hline \multicolumn{6}{|c|}{ Sabayés } \\
\hline Mayo 95 & 23,5 & 31,5 & 31,1 & 2,9 & $\overline{11,1}$ \\
\hline Abril 98 & 21,6 & 25,8 & 18,4 & 18,9 & 15,4 \\
\hline \multicolumn{6}{|c|}{ Arnás } \\
\hline Junio 95 & 19,4 & 59,2 & 19,9 & 1,4 & 0,0 \\
\hline Ago. 98 & 24,2 & 46,4 & 21,9 & 7,5 & 0,0 \\
\hline
\end{tabular}

Los coeficientes de escorrentía determinados a partir de las simulaciones de lluvia en toda el área de estudio oscilan entre $0 \%$ y $76 \%$, y la media alcanza el $28 \%$, demostrando así la alta variabilidad de la capacidad de infiltración, resultado de los procesos de bioturbación y la colonización de la superficie por líquenes. El valor medio de la producción de sedimento por experimento es de $16 \mathrm{~g} \mathrm{~m}^{-2}$. El coeficiente de escorrentía superficial medido con simulaciones de lluvia en ambientes relacionados con la par- 


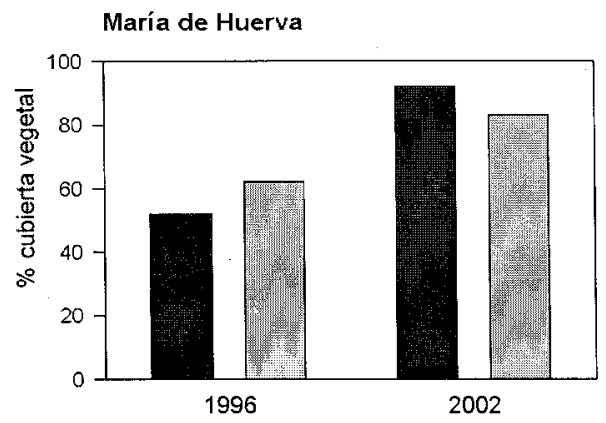

Figura 4. Resultados del análisis de la evolución de la cubierta vegetal y de la geomorfodinámica actual en María de Huerva, Depresión Central del Ebro.

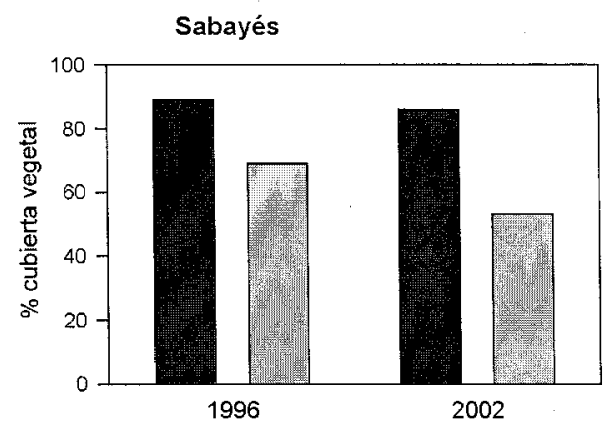

Figura 5. Resultados del análisis de la evolución de la cubierta vegetal y de la geomorfodinámica actual en Sabayés, Prepirineo.

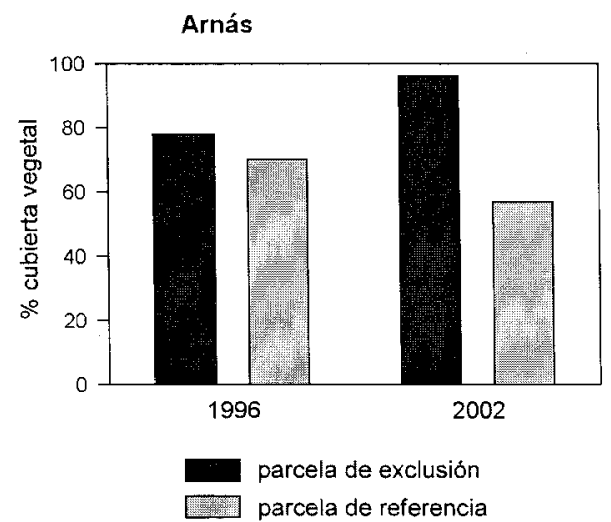

Figura 6. Resultados del análisis de la evolución de la cubierta vegetal y de la geomorfodinámica actual en Arnás, Pirineo Central. 
cela estudiada oscila entre 15 y 63\%, claramente más alto que la media total. Lo mismo puede observarse con las tasas de erosión, que alcanzan valores entre $22 \mathrm{y} 77 \mathrm{~g} \mathrm{~m}^{-2}$. A causa de la presión del ganado y de la actividad geomorfológica no existe una recolonización vegetal. La figura 7 muestra los resultados de las parcelas de control de pastoreo vecinas: al comienzo de los muestreos, la parcela pastoreada mostraba una cobertura vegetal un 10\% superior a la de la protegida. En octubre de 2002, la situación habia cambiado significativamente: a pesar de haberse producido un aumento de un $21 \%$ en el cubrimiento vegetal, la parcela protegida incrementó su cubierta vegetal en un $40 \%$.

\subsection{Prepirineo}

La parcela del Prepirineo se identifica con un viñedo abancalado abandonado hace 4 décadas y actualmente colonizado por aliagas. El riesgo de erosión de este ambiente se refleja en los altos coeficientes de escorrentía -media de 34\%- y en una erosión extremadamente intensa que ronda los $43 \mathrm{~g} \mathrm{~m}^{-2}$. Esta zona está caracterizada por un desceso generalizado de la cubierta vegetal de una media -de un 73 a un $61 \%$, a pesar de que las precipitaciones han sido en el periodo estudiado superiores a la media anual (Fig. 5, Tabla 1). En general se trata de una reducción de la 'cubierta densa', simultánea al aumento de la 'cubierta discontinua' y la 'cubierta baja'.

Los cambios de la geomorfodinámica muestran un aumento generalizado de la actividad de procesos. Hay que destacar el notable aumento de la 'erosión lineal' de un 3 a un 19\% (Tabla 2). Los surcos, de hasta $16 \mathrm{~m}$ de longitud, comienzan en los senderos diagonales del ganado para luego dejar éstos y seguir la pendiente. Estos senderos muestran coeficientes de escorrentia entre el $26 \%$ y el $66 \%$ y llegan a producir hasta $334 \mathrm{~g} \mathrm{~m}^{-2}$ de sedimento (la media de 5 simulaciones sigue siendo de $110 \mathrm{~g} \mathrm{~m}^{-2}$ ). El ganado amplía y profundiza estos senderos, al mismo tiempo que reduce la cubierta vegetal en sus bordes. En un 7\% de la superficie se puede observar 'erosión lineal' en áreas con una cubierta vegetal densa. Así, la evolución de los surcos tambien es posible en áreas con una cubierta vegetal cerrada. El análisis de las parcelas de control del ganado muestra un descenso de la cubierta vegetal de un 16\% en las superficies pastoreadas, mientras que las superficies protegidas mantienen su cubierta vegetal (Fig. 7).

\subsection{Pirineo Central}

La parcela muestreada en Arnás, un campo abandonado hace 30 años dividido en una zona con matorral denso de aliaga y otra con un pastizal de Brachypodium spec. muestra en el periodo estudiado un descenso leve $(5 \%)$ de la cubierta vegetal. La escorrentía varía entre $0 \%$ y $57 \%$ de la precipitación aplicada, y la media es del 19\%, relativamente alta si se tiene en cuenta que la cubierta vegetal es densa. Las tasas de erosión media son, en cambio, relativamente bajas y solo alcanzan $5 \mathrm{~g} \mathrm{~m}^{-2}$. 

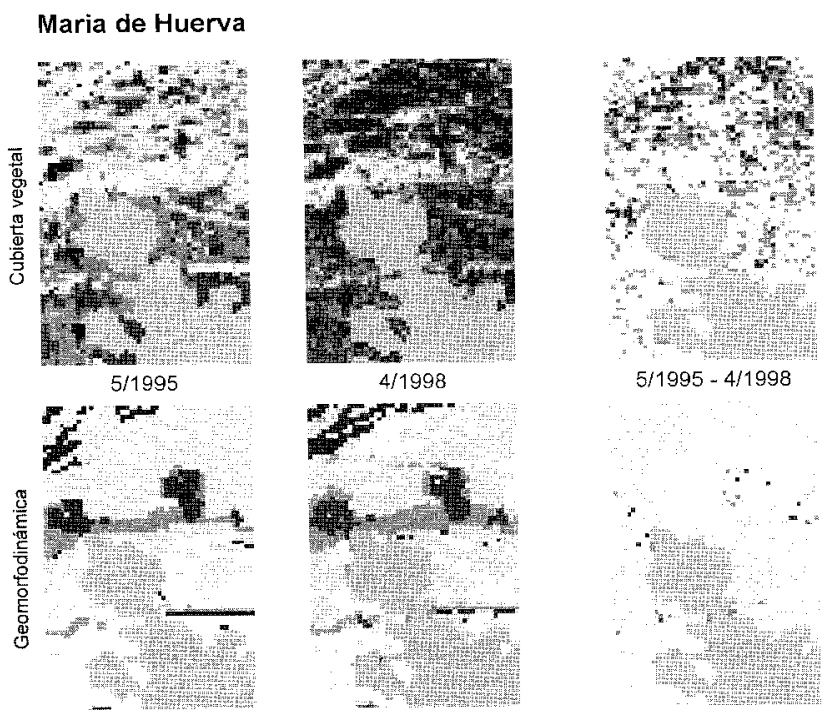

$5 / 1995-4 / 1998$

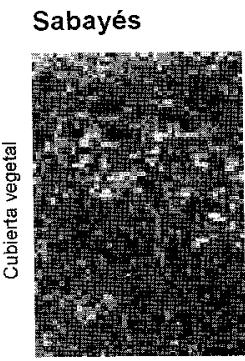

$5 / 1995$

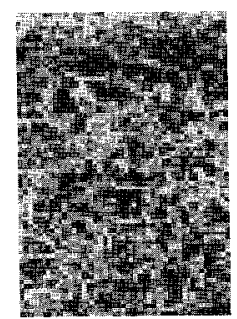

$4 / 1998$
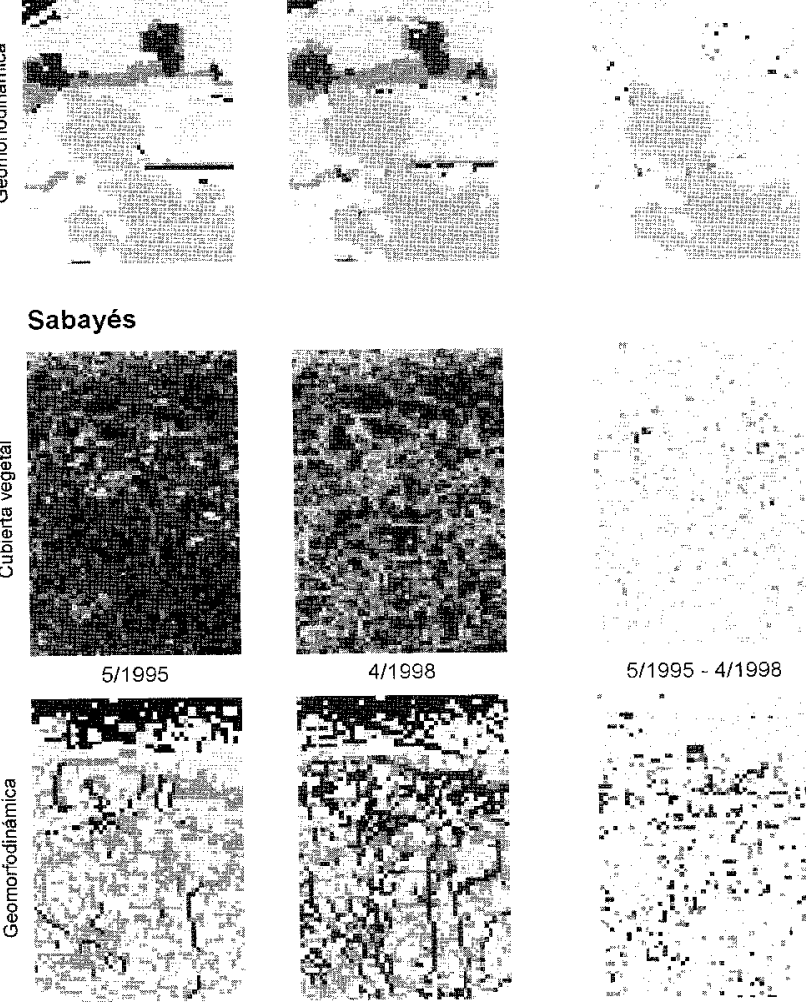

$5 / 1995-4 / 1998$

-
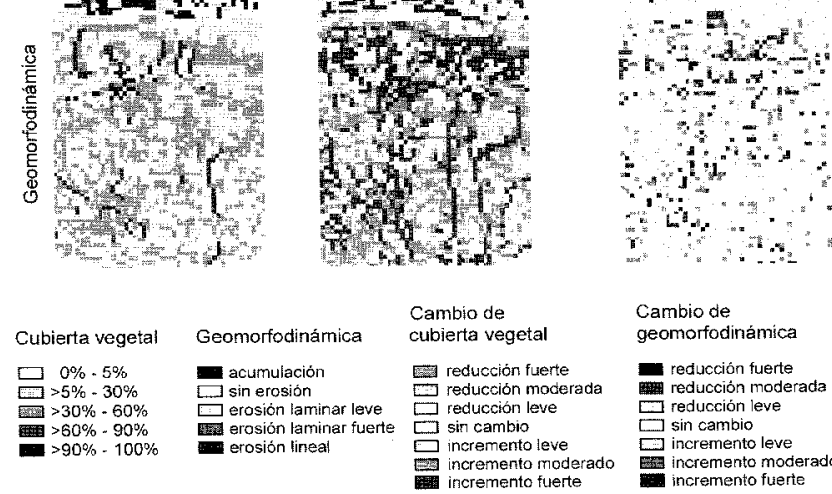

Cambio de

geomorfodinámica

- reducción fuerte

reducción moderada

品 reducción leve

Jin cambio

incremento leve

Figura 7. Evolución de la cubierta vegetal entre 1996 y 2002 en las parcelas de control de pastoreo (parcelas de exclusión y parcelas de referencia) en María de Huerva, Sabayés y Arnás. 
Tabla 3. Cambios de la cubierta vegetal y de la la geomorfodinámica actual en \% en las parcelas de María de Huerva, Sabayés y Arnás de 1995 a 1998.

\begin{tabular}{|c|c|c|c|c|c|c|c|}
\hline $\begin{array}{l}\text { Periodo } \\
1995-1998 \\
{[\%]}\end{array}$ & $\begin{array}{c}\text { Descenso } \\
\text { fuerte } \\
{[\%]}\end{array}$ & $\begin{array}{c}\text { Descenso } \\
\text { moderado } \\
{[\%]}\end{array}$ & $\begin{array}{c}\text { Descenso } \\
\text { leve } \\
{[\%]}\end{array}$ & $\begin{array}{c}\operatorname{Sin} \\
\text { cambios } \\
{[\%]}\end{array}$ & $\begin{array}{c}\text { Incremento } \\
\text { leve } \\
{[\%]}\end{array}$ & $\begin{array}{c}\text { Incremento } \\
\text { moderado } \\
{[\%]}\end{array}$ & $\begin{array}{c}\text { Incremento } \\
\text { fuerte }\end{array}$ \\
\hline \multicolumn{8}{|c|}{ Maria de Huerva } \\
\hline $\begin{array}{l}\text { Cubierta } \\
\text { vegetal }\end{array}$ & 0,0 & 0,2 & 3,1 & 17,3 & 28,2 & 18,5 & 6,4 \\
\hline $\begin{array}{l}\text { Geomorfo- } \\
\text { dinámica }\end{array}$ & 0,1 & 0,2 & 8,2 & 51,8 & 12,7 & 0,6 & 0,1 \\
\hline \multicolumn{8}{|c|}{ Sabayés } \\
\hline $\begin{array}{l}\text { Cubierta } \\
\text { vegetal }\end{array}$ & 1,1 & 12,1 & 31,2 & 41,9 & 12,1 & 1,3 & 0,3 \\
\hline $\begin{array}{l}\text { Geomorfo- } \\
\text { dinámica }\end{array}$ & 0,4 & 4,6 & 14,7 & 48,5 & 24,3 & 5,8 & 1,8 \\
\hline \multicolumn{8}{|c|}{ Arnás } \\
\hline $\begin{array}{l}\text { Cubierta } \\
\text { vegetal }\end{array}$ & 0,0 & 0,8 & 27,0 & 58,9 & 12,6 & 0,6 & 0,1 \\
\hline $\begin{array}{l}\text { Geomorfo- } \\
\text { dinámica }\end{array}$ & 0,0 & 1,6 & 11,2 & 66,0 & 18,9 & 2,1 & 0,3 \\
\hline
\end{tabular}

En Arnás se puede observar una distribución espacial mas contrastada que en la parcela de Sabayés, con un ligero aumento de las clases más extremas (Fig, 6). Así, al final del análisis, podemos observar una duplicación de las clases 'cubierta vegetal baja' y 'cubierta vegetal discontínua', al mismo tiempo que la 'cubierta vegetal cerrada' aumenta hasta alcanzar el 3,6\% de la superficie. En este caso tambien podemos identificar el pastoreo como factor determinante: la 'reducción moderada' de la cubierta vegetal está concentrada en el pastizal inferior y en los senderos del ganado (Fig. 6, Tabla 3). De forma semejante a la evolución observada en el Prepirineo, la reducción de la cubierta vegetal se concentra en las áreas que al principio del estudio mostraban una 'cubierta vegetal densa', mientras que las zonas con 'cubierta vegetal cerrada' se han expandido. En el caso de la geomorfodinámica se puede observar una tendencia contraria: especialmente destacable es la multiplicación por 5 de la superficie con 'erosión lineal', que está ligada a los senderos del ganado ya existentes en 1995 y que coalescen y ganan superficie (Fig. 6, Tabla 2). En la parte inferior de la parcela se concentran las zonas con 'erosión laminar fuerte'. Sólamente aquellas zonas que no pueden ser alcanzadas por el ganado, a causa del matorral de aliaga denso, puede aumentar la cubierta vegetal y reducirse la geomorfodinámica. La producción de escorrentía es muy variable, pero en general baja, tanto en el pastizal como en las zonas con pedregosidad superficial. En los senderos del ganado los valores de escorrentía y erosión destacan, con $57 \%$ y $45 \mathrm{~h} \mathrm{~m}^{-2}$ respectivamente, siguiendo la misma tendencia que las otras áreas de estudio. La parcela de control del pastoreo 
Tabla 4. Simulaciones de lluvia en las zonas estudiadas de María de Huerva, Sabayés y Arnás. Intensidad: $40 \mathrm{~mm} \mathrm{~h}^{-1}$, duración: 30 min (Langer et al., 1999; Ries et al., 2000)

\begin{tabular}{|c|c|c|c|c|c|c|c|}
\hline Nr. & ID & Tipo de superficie & $\begin{array}{c}\text { pendiente } \\
{\left[\left[^{\circ}\right]\right.}\end{array}$ & $\begin{array}{c}\text { Cubierta } \\
\text { vegetal } \\
{[\%]}\end{array}$ & $\begin{array}{c}\text { Cubierta } \\
\text { de piedras } \\
{[\%]}\end{array}$ & $\begin{array}{c}\text { Coeficiente } \\
\text { de escorrentía } \\
{[\%]}\end{array}$ & $\begin{array}{l}\text { Erosión } \\
{\left[\mathrm{g} \mathrm{m}^{-2}\right]}\end{array}$ \\
\hline \multicolumn{8}{|c|}{ María de Huerva } \\
\hline 1 & 421 & Arbusto & 15 & 95 & - & 4 & 1 \\
\hline 2 & 546 & Herbácea con rizoma & 5 & 95 & - & 23 & 7 \\
\hline 3 & 551 & Herbácea con rizoma & 9 & 80 & - & 76 & 22 \\
\hline 4 & 582 & Líquenes & 2 & 80 & - & 0 & 0 \\
\hline 5 & 550 & Liquenes & 5 & 70 & - & 72 & 13 \\
\hline 6 & 584 & Líquenes & 2 & 70 & - & 0 & 0 \\
\hline 7 & 548 & Líquenes & 4 & 60 & - & 67 & 37 \\
\hline 8 & 433 & Líquenes & 5 & 50 & - & 3 & 0 \\
\hline 9 & 549 & Coprolitos & 6 & 50 & - & 6 & 9 \\
\hline 10 & 547 & Coprolitos & 5 & 40 & - & 29 & 17 \\
\hline 11 & 552 & Coprolitos & 13 & 40 & - & 59 & 35 \\
\hline 12 & 422 & Herbácea con rizoma & 15 & 40 & - & 27 & 10 \\
\hline 13 & 418 & Costra ectáfica & 2 & 3 & - & 0 & 0 \\
\hline 14 & 423 & Coprolitos & 7 & 25 & - & 0 & 0 \\
\hline 15 & 585 & Borde bancal & 15 & 3 & - & 63 & 77 \\
\hline 16 & 202 & Sendero ganado & 3 & 1 & - & 15 & 22 \\
\hline 17 & 419 & Sendero ganado & 10 & 1 & - & 37 & 22 \\
\hline Media & & & & & & 28 & 16 \\
\hline \multicolumn{8}{|c|}{ Sabayés } \\
\hline 1 & 560 & Herbáceas, prado & 10 & 95 & 5 & 37 & 4 \\
\hline 2 & 561 & Arbusto & 23 & 95 & 5 & 2 & 1 \\
\hline 3 & 426 & Arbusto & 12 & 90 & 5 & 0 & 0 \\
\hline 4 & 427 & Herbáceas, prado & 15 & 80 & 10 & 22 & 1 \\
\hline 5 & 206 & Arbusto & 7 & 65 & 15 & 0 & 0 \\
\hline 6 & 205 & Herbáceas, prado & 12 & 60 & 35 & 0 & 0 \\
\hline 7 & 559 & Piedras superficiales & 13 & 40 & 45 & 62 & 28 \\
\hline 8 & 562 & Herbáceas, prado & 14 & 35 & 35 & 96 & 29 \\
\hline 9 & 555 & Sendero ganado & 21 & 25 & 5 & 54 & 116 \\
\hline 10 & 424 & Borde bancal & 25 & 20 & 10 & 17 & 26 \\
\hline 11 & 425 & Senclero ganado & 13 & 20 & 5 & 26 & 4 \\
\hline 12 & 556 & Sendero ganado & 25 & 15 & 10 & 66 & 334 \\
\hline 13 & 557 & Sendero ganado & 20 & 15 & 20 & 60 & 42 \\
\hline 14 & 204 & Piedras superficiales & 8 & 5 & 85 & 16 & 4 \\
\hline 15 & 558 & Sendero ganado & 15 & 2 & 15 & 59 & 55 \\
\hline Media & & & & & & 34 & 43 \\
\hline \multicolumn{8}{|c|}{ Arnás } \\
\hline 1 & 210 & Herbáceas, prado & 6 & $\overline{100}$ & 0 & 24 & 2 \\
\hline 2 & 421 & Herbáceas, prado & 12 & 100 & 0 & 27 & 0 \\
\hline 3 & 547 & Herbáceas, prado & 5 & 75 & 15 & 0 & 0 \\
\hline 4 & 548 & Hebáceas, prado & 10 & 65 & 25 & 35 & 5 \\
\hline 5 & 420 & Piedras superficiales & 10 & 50 & 50 & 35 & 4 \\
\hline 6 & 549 & Arbusto & 8 & 50 & 40 & 20 & 11 \\
\hline 7 & 550 & Piedras superficiales & 10 & 40 & 50 & 2 & 2 \\
\hline 8 & 422 & Piedras superficiales & 8 & 30 & 60 & 8 & 2 \\
\hline 9 & 211 & Herbáceas, prado & 7 & 25 & 35 & 0 & 0 \\
\hline 10 & 212 & Piedras superficiales & 4 & 20 & 60 & 1 & 0 \\
\hline 11 & 551 & Sendero ganado & 14 & 20 & 40 & 57 & 45 \\
\hline Media & & & & & & 19 & 6 \\
\hline
\end{tabular}


demuestra de forma clara la influencia del ganado en la evolucion de la cubierta vegetal. Mientras que la parcela accesible a los animales sufre un descenso de la cubierta vegetal del 13\%, la parcela protegida la incrementa hasta un 18\%. Durante el periodo estudiado, entre 1996 y 2002, la cubierta vegetal ha llegado a colonizar toda la superficie.

\section{Conclusiones}

El muestreo contínuo y los estudios experimentales demuestran que el pastoreo frena significativamente la sucesión de la vegetación. Es más, en el Pirineo Central y en el Prepirineo la carga ganadera es la causa de un descenso generalizado de la cubierta vegetal y de su diferenciación espacial. A su vez, la dinámica geomorfológica se incrementa en todas las zonas pastoreadas. En especial los surcos desarrollados en los senderos de los animales, aumentan intensamente. Precisamente son los senderos creados y recrecidos por el pisoteo del ganado las zonas que han de considerarse mas activas en lo que se refiere a la geomorfodinámica. En ellos se generan las tasas mas altas de escorrentía y de erosión. La cubierta vegetal podría recuperarse con la exclusión completa del ganado; la flora herbácea se recuperaría y en pocos años cabría esperar el cubrimiento completo del suelo. Estos resultados ponen en entredicho las subvenciones de la política agraria europea al ganado ovino, a causa de sus consecuencias sobre la evolución de la vegetación y la geomorfodinámica actual.

Para la reducción de la escorrentía superficial y la erosion en estas áreas, ya degradadas por su aprovechamiento secular, sería recomendable limitar el pastoreo de forma generalizada.

\section{Agradecimientos}

El proyecto EPRODESERT ha sido financiado por la Deutsche Forschungsgemeinschaft (DFG, Ri 375/13). M. Seeger e I. Marzolff, además, gozaron de becas del Estado de BadenWürttemberg/Alemania (GraFöG). Los autores queremos expresar nuestra gratitud a todos aquellos que nos han apoyado y alentado en nuestras investigaciones por tierras aragonesas.

Los resultados obtenidos en el presente artículo completan los publicados en la revista de lengua alemana Geographische Rundschau (05/2003). 


\section{Bibliografía}

Braun-Blanquet, J., de Bolòs, O. (1987): Las comunidades vegetales de la Depresión del Ebro y su dinamismo. Reeditado 1987. Delegación de Medio Ambiente, Ayuntamiento de Zaragoza. Ins Spanische übersetzte Neuauflage des 1957 erschienenen: Les groupements vegeteaux du Bassin Moyen de l'Ebre et leur dinamisme, Anales Estación Experimental Aula Dei, 5 (1-4), Zaragoza, 278 pp.

Gallart, F.; Llorens, P., Latron, J. (1994): Studying the role of old agricultural terraces on runoff generation in a small Mediterranean mountainous basin. Journal of Hydrology, 159, p. 291-303.

García-Ruiz, J. M. \& Lasanta, T. (1993): Landuse conflicts as a result of land-use changes in the Spanish Pyrenees. Mountain Research and Development, 13 (3), p. 295304.

García-Ruiz, J.M., Valero-Garcés, B.L. (1998): Historical geomorphic processes and human activities in the Central Spanish Pyrenees. Mountain Research and Development, 18 (4), p. 309-320.

Harrer, S. (1996b): Zum Einfluf der Landnutzung und des Landnutzungswandels auf die Vegetation zweier mediterraner Bereiche. Unveröffentl. Zulassungsarbeit, Universität Freiburg, Institut für Physische Geographie; Freiburg i. Br., $107 \mathrm{~S}$.

Ibarra Benlloch, P., Martínez Ferrer, J. (1995a): Vegetación potencial. Atlas de Geografia de Aragon 1.3.1., Zaragoza.

Llorens P., Queralt, I., Plana, F., Gallart, F. (1997): Studying solute and particulate sediment transfer in a small Mediterranean mountainous catchment subject to land abandonment. Earth Surface Processes and Landforms, 22, p. 1027-1035.

Langer M., Langhagen, R., Neeb, S., Pfahls, Ch., Weise, M. (1999): Oberflächenabfluß, Suspensions-, Lösungsfracht und Infiltration. 111 Niederschlagssimulationen mit einer mobilen Kleinberegnungsanlage. In: Ries. J.B. [Hrsg.]: EPRODESERT - Bodenwasserhaushalt und aktuelle Geomorphodynamik auf Brachflächen in Aragón/Spanien. APT-Bericbte, Heft 10, Institut für Physische Geographie, Universität Freiburg i. Br., S., p. 79-103, Freiburg.

Lasanta, T., Garcia-Ruiz, J.M., Pérez-Rontomé, C., Sancho-Marcén, C. (2000): Runoff and sediment yield in a semi-arid environment. The effect of land management after farmland abandonment. Catena, 38, p. 265-278.

Lucht, A. (1998): Transformationsprozesse eines traditionellen Agrarsystems im spannungsfeld wechselnder sozialer, wirtschaftlicher und politischer Rahmenbedingungen. Das Beispiel der spanischen Pyrenäengemeinde Borau, Aragón. In: Drescher, A. \& Ries, J. B. [Hrsg.]: Aktuelle geographische Forschung in Andalusien und Aragonien. APT-Berichte, Heft 8; Institut für Physische Geographie, Universität Freiburg i. Br., S., p. 87-107. Freiburg.

Marzolff, I. (1999): Großmaßstäbige Fernerkundung mit einem unbemannten Heilsluftzeppelin für GIS-gestütztes Monitoring von Vegetationsentwicklung und Geomorphodynamik in Aragón (Spanien). Freiburger Geographische Hefte, 57, 226 S. + 99 S. Kartenanhang; Freiburg. 
Monserrat i Martí, J.M. (1986): Flora y Vegetación de la Sierra de Guara (Prepirineo Aragonés). Naturaleza en Aragón, 1, Zaragoza, $334 \mathrm{pp}$.

Seeger, M. (2001): Boden und Bodenwasserhaushalt als Indikatoren der Landdegradierung auf extensivierten Nutzflächen in Aragón / Spanien. Freiburger Geographiscbe Hefte, 63, Freiburg i. Br., $184+$ $30 \mathrm{~S}$.

Ries, J.B. (2002): Geomorphodynamics on fallow land and abandoned fields in the Ebro Basin and the Pyrenees - Monitoring of processes and development. Z.GeOmorph. N.F., 127, p. 21-45.

Ries, J.B., Langer, M., Rehberg, C. (2000): Experimental investigations on water and wind erosion on abandoned fields and arable land in the Central Ebro Basin,
Aragón/Spain. Z. Geomorph. N.F., Suppl. 121, p. 91-108.

Rivas-Martínez, S. (1987): Mapa de series de vegetación de España 1:400.000 y menoría, 208 pp., Madrid.

Ruiz-Flãno, P. (1993): Procesos de erosión en campos abandonados del Pirineo. El ejemplo del valle de Aisa. Geoforma Ediciones, No. 4, 191 pp., Logroño.

Sala, M., Rubio, J.L., García-Ruiz, J.M. leds.] (1991): Soil Erosion Studies in Spain. Logroño, $228 \mathrm{pp}$.

Weiler, F., Poschacher, R. (2002): Erhebung der Bestände an Schafen und Ziegen im Dezember 2001 sowie Erzeugungsvorausschätzungen 2002. EUROSTAT, Statistik kurz gefasst, Landwirtschaft und Fischerei, Thema 5-12/2002 Landwirtschaft, S. 1-3. 